\title{
Sufficient conditions on existence of solution for nonlinear fractional iterative integral equation
}

\author{
Faten H. M. Damag, Adem Kılıçman* \\ Department of Mathematics and Institute for Mathematical Research, University Putra Malaysia, 43400 Serdang, Selangor, Malaysia.
}

Communicated by A. Atangana

\begin{abstract}
In this article, we study nonlinear quadratic iterative integral equations and establish sufficient conditions for the existence of Volterra solutions for fractional iterative integral equations and solvency in Banach space and $C_{\ell, \beta}$. In the present work we use the principle of contraction, Schaefer's fixed point theorem and the non-expansive operator method as essential tools. In this study we consider Riemann-Liouville differential operator and prove some related theorems, further provide an example as an application. (C)2017 all rights reserved.
\end{abstract}

Keywords: Fractional integral equation, existence of solution, Schaefer's fixed point theorem, non-expansive operator. 2010 MSC: 39A12, 37N25, 35E15.

\section{Introduction}

The mathematical modeling in a biological phenomena has getting increasingly important since it has an impact in our live. Thus there are many researchers in the literature who studied and have dealt this type of problems, for example, see ([2, 5, 7, 8, 11, 12, 15, 16, 21, 22]).

During the process of modeling one can generate an integral equation and formulate related theorems. Further related applied problems with integral equations are also growing very rapidly with the aid of various tools such as functional analysis, topology and the theory of fixed point. In particular, integral equations (quadratic type) have many beneficial applications in real-world problems. For instance, the integral equations of the quadratic type are very applicable to the radioactive transference theory, theory of the kinetics of gases, the neutron transport theory, theory of traffic, and as well as theory of queuing [9]. It is also recognized that nonlinear quadratic iterative integral equations are often used in biological applications. The fixed point theorems which are utilized in the nonlinear functional analysis generally used to obtain existence of solutions concerning the investigative functional-operator equations.

In this present work, we are also interested in the mathematical biological model, and we study the nonlinear quadratic iterative integral equations and establish sufficient conditions on existence of

\footnotetext{
*Corresponding author

Email addresses: faten_212326@hotmail.com (Faten H. M. Damag), akilic@upm.edu.my (Adem Kılıçman)
} 
solutions for the Volterra type fractional iterative integral equation and solvency in Banach space $C_{\ell, \beta}$. In our study we employ non-expansive operator technique as essential tools, and include the principle of contraction and Schaefer's fixed point theorem. The new result generalizes the previous results, see $[1,3,10,14,18]$. Further we also establish some related existence results for some integral equations with a modified argument of fractional order, see the details in [6].

In the progress of the study we need some definitions, notations, and preliminary data that we will use in the development of the paper, see $[13,17,19,20]$. Let $C(I, R)$ be Banach space of all continuous functions from $I$ to $R$ and equipped with the norm

$$
\|w\|:=\sup \{|w(s)|: s \in I\} .
$$

Then recall some important theorems related to fixed point which are used in the paper. These theorems may be found in the following papers $[3,18]$.

Definition 1.1. The integral operator is defined as

$$
\mathrm{I}_{\mathrm{a}}^{\alpha} \psi(\mathrm{s})=\frac{1}{\Gamma(\alpha)} \int_{0}^{s} \frac{\psi(\beta)}{(s-\beta)^{1-\alpha}} \mathrm{d} \beta,
$$

where $\alpha>0$.

Definition 1.2. The fractional differentiation operator (Riemann-Liouville) is defined as

$$
D_{0}^{\alpha} \psi(s)=\frac{1}{\Gamma(\iota-\alpha)}\left[\frac{d}{d s}\right]^{\imath} \int_{0}^{s} \frac{\psi(\beta)}{(s-\beta)^{\alpha-\imath+1}} d \beta, \quad(\iota-1) \leqslant \alpha<\iota,
$$

where $\iota$ is a whole number and $\alpha$ is a real number.

Definition 1.3. The fractional differentiation operator (Caputo) is defined as

$$
D_{0}^{\alpha} \psi(s)=\frac{1}{\Gamma(\iota-\alpha)} \int_{0}^{s} \frac{\psi^{(\imath)}(\beta)}{(s-\beta)^{\alpha-\imath+1}} d \beta, \quad(\iota-1) \leqslant \alpha<\iota,
$$

where $\iota$ is a whole number and $\alpha>0$ is a real number.

Definition 1.4. Let $(W, d)$ be a metric space and $Q \subseteq C(W, R)$. We say $Q$ is a equicontinuous if for all $\epsilon>0$ there is $\delta>0$ so that for all $g \in Q, w, a \in W$ then

$$
\mathrm{d}(w, \mathrm{a})<\delta \Rightarrow|g(w)-g(\mathrm{a})|<\epsilon .
$$

Theorem 1.5. Let $(\mathrm{W}, \mathrm{d})$ be a compact metric space and $\mathrm{Q} \subseteq \mathrm{C}(\mathrm{W}, \mathrm{R})$. Then, $\mathrm{Q}$ is compact if and only if $\mathrm{Q}$ is equicontinuous, closed and bounded. This theorem is called the Arzelá-Ascoli theorem.

Theorem 1.6. Let $\mathrm{E}$ be a continuous and compact mapping in a Banach space $\mathrm{W}$ into itself, such that the set

$$
\{w \in W: w=\sigma E w, \text { for some } 0 \leqslant \sigma \leqslant 1\}
$$

$\mathrm{E}$ is bounded. Then $\mathrm{E}$ has a fixed point. This theorem is known as the Schaefer theorem.

Theorem 1.7. Let $\mathrm{W}$ be a Banach space and $\mathrm{Q} \neq \emptyset$ subset of $\mathrm{W}$, compact, and convex. Now, if $\mathrm{T}: \mathrm{Q} \rightarrow \mathrm{Q}$ is a continuous operator, then $\mathrm{T}$ has at least one fixed point in $\mathrm{Q}$. This theorem is also called the Schaefer theorem.

\section{Main Results}

In this section, we propose and find some outcomes concerning the existing solution of iterative integral equations of fractional order. 
Let us consider the following nonlinear fractional iterative integral equation of quadratic type:

$$
v(s)=g(s, v(s))+f(s, v(s)) \int_{0}^{s} \frac{(s-u)^{(\beta-1)}}{\Gamma(\beta)} K(u, v(u), v(v(u))) d u .
$$

Let $\mathrm{I}=[0, \mathrm{~b}], \mathrm{T}=\max \{0, \mathrm{~b}\}$, and

$$
C_{\ell, \beta}=\left\{v \in C(I, I):\left|v\left(u_{1}\right)-v\left(u_{2}\right)\right| \leqslant \ell \cdot \frac{\left|u_{1}-u_{2}\right|^{\beta}}{\Gamma(\beta+1)}, \forall u_{1}, u_{1} \in R_{+}\right\}, \ell>0 .
$$

Our first finding is based on Banach fixed point theorem.

Theorem 2.1. Suppose the following conditions are achieved

$\left(b_{1}\right) K: R_{+} \times R \times R \rightarrow R, f, g: R_{+} \times R \rightarrow R$ functions of continuous are given;

$\left(b_{2}\right) \exists \ell_{1}>0$, so that

$$
|g(s, v)-g(s, w)| \leqslant \ell_{1}|v-w|, \forall s \in I, v, w \in R
$$

$\left(b_{3}\right) \exists \ell_{2}>0$ so that

$$
|\mathrm{f}(\mathrm{s}, v)-\mathrm{f}(\mathrm{s}, w)| \leqslant \ell_{2}|v-w|, \forall s \in \mathrm{I}, v, w \in \mathrm{R}
$$

$\left(\mathrm{b}_{4}\right) \exists \ell_{3}>0$ so that

$$
|k(s, v, v(v))-k(s, w, w(w))| \leqslant \ell_{3}[|v-w|+|v(v)-w(w)|] \leqslant \ell_{3}(\ell+2)|v-w|, \forall s \in I, v, w \in R .
$$

If

$$
\sup _{s \geqslant 0}\left\{\ell_{1}+\ell_{2} \cdot \int_{0}^{s} \frac{(s-u)^{(\beta-1)}}{\Gamma(\beta)}|K(u, v(u), v(v(u)))| d u+\ell_{3}(2+\ell) \frac{T^{\beta}}{\Gamma(\beta+1)}|f(s, v(s))|\right\} \leqslant k<1, \forall t \in I,
$$

then equation (2.1) has a unique solution on $\mathrm{I}$.

Proof. The transformation of Eq. (2.1) in a fixed-point problem: Consider the operator:

$$
\mathrm{G}: \mathrm{C}(\mathrm{I}, \mathrm{R}) \rightarrow \mathrm{C}(\mathrm{I}, \mathrm{R})
$$

defined by

$$
\mathrm{G}(v)(s)=g(s, v(s))+f(s, v(s)) \int_{0}^{s} \frac{(s-u)^{(\beta-1)}}{\Gamma(\beta)} \mathrm{K}(\mathrm{u}, v(\mathrm{u}), v(v(u))) d u
$$

The fixed point of the operator $\mathrm{G}$ is a solution of Eq. (2.1). We will use the principle of Banach contraction mapping to demonstrate that $\mathrm{G}$ has a fixed point.

Let $v, w \in \mathrm{C}[\mathrm{I}, \mathrm{R}]$

$$
\begin{aligned}
& |(G v)(u)-(G w)(u)| \\
& \leqslant|g(s, v(s))-g(s, w(s))|+\mid f(s, v(s)) \int_{0}^{s} \frac{(s-u)^{(\beta-1)}}{\Gamma(\beta)} K(u, v(u), v(v(u))) d u \\
& \quad-f(s, v(s)) \int_{0}^{s} \frac{(s-u)^{(\beta-1)}}{\Gamma(\beta)} K(u, w(u), w(w(u))) d u \\
& \quad+f(s, v(s)) \int_{0}^{s} \frac{(s-u)^{(\beta-1)}}{\Gamma(\beta)} K(u, w(u), w(w(u))) d u \\
& \quad-f(s, w(s)) \int_{0}^{s} \frac{(s-u)^{(\beta-1)}}{\Gamma(\beta)} K(u, w(u), w(w(u))) d u \mid
\end{aligned}
$$




$$
\begin{aligned}
& \leqslant \ell_{1}|v(s)-w(s)| \\
& +|f(s, v(s))| \cdot \int_{0}^{s}\left|\frac{(s-u)^{(\beta-1)}}{\Gamma(\beta)} K(u, v(u), v(v(u)))-K(u, w(u), w(w(u)))\right| d u \\
& +|f(s, v(s))-f(s, w(s))| \cdot \int_{0}^{s} \frac{(s-u)^{(\beta-1)}}{\Gamma(\beta)} K(u, w(u), w(w(u))) d u \\
& \leqslant \ell_{1}|v(s)-w(s)|+\ell_{3}|f(s, v(s))| \cdot \int_{0}^{s} \mid \frac{(s-u)^{(\beta-1)}}{\Gamma(\beta)}[|v(u)-w(u)|+|v(v(u))-w(w(u))|] d u \\
& +\ell_{2}|v(s)-w(s)| \cdot \int_{0}^{s} \frac{(s-u)^{(\beta-1)}}{\Gamma(\beta)}|K(u, w(u), w(w(u)))| d u \\
& \leqslant \ell_{1}|v(s)-w(s)| \\
& +\ell_{3}|f(s, v(s))| \cdot \int_{0}^{s} \mid \frac{(s-u)^{(\beta-1)}}{\Gamma(\beta)}[|v(s)-w(s)|+\ell|v(s)-w(s)|+|v(w(s))-w(w(s))|] d u \\
& +\ell_{2}|v(s)-w(s)| \cdot \int_{0}^{s} \frac{(s-u)^{(\beta-1)}}{\Gamma(\beta)} \mathrm{K}(u, w(u), w(w(u))) d u \\
& \leqslant \ell_{1}|v(s)-w(s)|+\ell_{3}[2+\ell]|f(s, v(s))| \cdot \int_{0}^{s} \frac{(s-u)^{(\beta-1)}}{\Gamma(\beta)}|v(s)-w(s)| d u \\
& +\ell_{2}|v(s)-w(s)| \cdot \int_{0}^{s} \frac{(s-u)^{(\beta-1)}}{\Gamma(\beta)} K(u, w(u), w(w(u))) d u .
\end{aligned}
$$

Then

$$
\begin{aligned}
\|G v-G w\| \leqslant & {\left[\ell_{1}+\ell_{2} \cdot \int_{0}^{s} \frac{(s-u)^{(\beta-1)}}{\Gamma(\beta)}|K(u, w(u), w(w(u)))| d u\right.} \\
& \left.+\ell_{3}(2+\ell) \frac{T^{\beta}}{\Gamma(\beta+1)}|f(s, v(s))|\right]\|v(s)-w(s)\| .
\end{aligned}
$$

Therefore, $\mathrm{G}$ is a contraction. As a result of the Banach fixed point theorem, we can deduce that $\mathrm{G}$ has a fixed point and the solution to Eq. (2.1).

The second outcome is based on the Schaefer's fixed point theorem.

Theorem 2.2. Suppose the following conditions are satisfied.

$\left(a_{1}\right)$ The functions

$$
f, g: R_{+} \times R \rightarrow R \text { and } K: R_{+} \times R \times R \rightarrow R
$$

are continuous;

$\left(a_{2}\right)$ if $\ell$ is the Lipschitz constant and satisfies (2.2), then

$$
\int_{0}^{s_{1}} \frac{\left(s_{1}-u\right)^{(\beta-1)}}{\Gamma(\beta)} K(u, v(u), v(v(u))) d u-\int_{0}^{s_{2}} \frac{\left(s_{2}-u\right)^{(\beta-1)}}{\Gamma(\beta)} K(u, v(u), v(v(u))) d u \leqslant \ell \frac{\left|s_{1}-s_{2}\right|^{\beta}}{\Gamma(\beta+1)},
$$

for each $\mathrm{s}_{1}, \mathrm{~s}_{2} \in \mathrm{I}$ and $v \in \mathrm{R}$ and

$$
\Xi=\max _{s \in I}\left\{\left|f(s, v(s)) \cdot \int_{0}^{s} \frac{(s-u)^{(\beta-1)}}{\Gamma(\beta)} K(u, v(u), v(v(u))) d u\right|\right\}, \forall s \in I, v \in R
$$

$\left(a_{3}\right)$ there are constants $n, N, \zeta \in R$ so that

$$
|\mathrm{g}(s, v)-\mathrm{g}(\mathrm{s}, z)| \leqslant n|v-z| \text {, for each } v, z \in \mathrm{R} \text { and } s \in \mathrm{I},
$$




$$
\begin{gathered}
|g(s, v)-g(u, v)| \leqslant N \cdot \ell \frac{|s-u|^{\beta}}{\Gamma(\beta+1)}, \text { and for all } s, u \in I, v \in R, \\
|g(s, v)| \leqslant \zeta, \text { for all } s \in I, v \in R
\end{gathered}
$$

$\left(a_{4}\right)$ there are constants $m, M \in R$ so that

$$
\begin{aligned}
& |f(s, v)-f(s, z)| \leqslant m|v-z|, \text { for each } v, z \in R \text { and } s \in I, \\
& |f(s, v)-f(u, v)| \leqslant M . \ell \frac{|s-u|^{\beta}}{\Gamma(\beta+1)}, \text { and for all } s, u \in I, v \in R
\end{aligned}
$$

$\left(a_{5}\right)$ there is a constant $k \in[0,1]$ so that

$$
\sup _{s \in I}\left\{n+N+(m+M) \int_{0}^{s} \frac{(s-u)^{(\beta-1)}}{\Gamma(\beta)}|K(u, v(u), v(v(u)))| d u+|f(s, v(s))|\right\} \leqslant k .
$$

Then there is a solution in $\mathrm{C}_{\ell, \beta}$ for the equation (2.1).

Proof. It is well known (see [4, Lemma 1]) that $C_{\ell, \beta} \neq \emptyset$ is convex, on the other hand, it is a subset of Banach space $(C[a, b],\|\|$.$) (where \|$.$\| is the usual supreme norm) and compact.$

Presently, we transform the equation (2.1) into problem of fixed point.

Now consider the integral operator:

$$
\mathrm{G}: \mathrm{C}_{\ell, \beta} \rightarrow \mathrm{C}(\mathrm{I}, \mathrm{R})
$$

and defined as

$$
(\mathrm{G} v)(\mathrm{s})=\mathrm{g}(\mathrm{s}, v(\mathrm{~s}))+\mathrm{f}(\mathrm{s}, v(\mathrm{~s})) \int_{0}^{\mathrm{s}} \frac{(\mathrm{s}-\mathrm{u})^{(\beta-1)}}{\Gamma(\beta)} \mathrm{K}(\mathrm{u}, v(\mathrm{u}), v(v(\mathrm{u}))) \mathrm{du}
$$

Then it is clear that the operator of fixed points $G$ is a solution for the equation (2.1). We shall use Schaefer's fixed point theorem to demonstrate that $\mathrm{G}$ has a fixed point.

Phase $1 . \mathrm{G}$ is continuous.

Let $\left\{v_{j}\right\}$ be o sequence so that $v_{j} \rightarrow v$ in $\mathrm{C}(\mathrm{I}, \mathrm{R})$. Therefore for all $s \in \mathrm{I}$

$$
\begin{aligned}
\left|G\left(v_{j}(s)\right)-G(v)(s)\right| \leqslant & \left|g\left(s, v_{j}(s)\right)-g(s, v(s))\right| \\
& +\left|f\left(s, v_{j}(s)\right)\right| \cdot \int_{0}^{s} \frac{(s-u)^{(\beta-1)}}{\Gamma(\beta)}\left[K\left(u, v_{j}(u), v_{j}\left(v_{j}(u)\right)\right)-K(u, v(u), v(v(u)))\right] d u \\
& +\left|f\left(s, v_{j}(s)\right)-f(s, v(s))\right| \cdot \int_{0}^{s} \frac{(s-u)^{(\beta-1)}}{\Gamma(\beta)} K(u, v(u), v(v(u))) d u .
\end{aligned}
$$

Since $\mathrm{g}, \mathrm{K}$ and $\mathrm{f}$ are continuous functions, we get the following

$$
\left\|\mathrm{G} v_{j}-\mathrm{G} v\right\| \rightarrow 0 \text {, as } j \rightarrow \infty .
$$

Phase 2. $G$ is a bounded map in $C_{\ell, \beta}$ into bounded sets in $C(I, R)$. Hence, just to prove for any $\mu>0$, there is a positive constant $L$ so that for all $v \in A_{\mu}=\left\{v \in C_{\ell, \beta}:\|v\| \leqslant \mu\right\}$, we have $\|G v\| \leqslant$ L. By $\left(a_{3}\right)-\left(a_{4}\right)$ we get for all $s \in \mathrm{I}$.

$$
|(G v)(s)| \leqslant|g(s, v(s))|+\left|f(s, v(s)) \int_{0}^{s} \frac{(s-u)^{(\beta-1)}}{\Gamma(\beta)} K(u, v(u), v(v(u))) d u\right| \leqslant \zeta+\Xi .
$$

Hence

$$
\|\mathrm{G} v\| \leqslant \zeta+\Xi:=\mathrm{L}
$$


Phase 3. G maps bounded sets into equicontinuous sets of $C_{\ell, \beta}$.

$$
\begin{aligned}
& \mid(\mathrm{G} v)\left(s_{1}\right)-\left(\mathrm{G} v\left(s_{2}\right) \mid\right. \\
& \leqslant\left\|g\left(s_{1}, v\left(s_{1}\right)\right)|-| g\left(s_{2}, v\left(s_{2}\right)\right)\right\| \\
& +\mid f\left(s_{1}, v\left(s_{1}\right)\right) \cdot \int_{0}^{s_{1}} \frac{\left(s_{1}-u\right)^{(\beta-1)}}{\Gamma(\beta)} K(u, v(u), v(v(u))) d u \\
& -f\left(s_{2}, v\left(s_{2}\right)\right) \cdot \int_{0}^{s_{2}} \frac{\left(s_{2}-u\right)^{(\beta-1)}}{\Gamma(\beta)} K(u, v(u), v(v(u))) d u \mid \\
& \leqslant\left|g\left(s_{1}, v\left(s_{1}\right)\right)-g\left(s_{2}, v\left(s_{1}\right)\right)\right|+\left|g\left(s_{2}, v\left(s_{1}\right)\right)-g\left(s_{2}, v\left(s_{2}\right)\right)\right| \\
& +\left|f\left(s_{1}, v\left(s_{2}\right)\right)-f\left(s_{2}, v\left(s_{1}\right)\right)\right| \int_{0}^{s_{1}} \frac{\left(s_{1}-u\right)^{(\beta-1)}}{\Gamma(\beta)} K(u, v(u), v(v(u))) d u \\
& +\left|f\left(s_{2}, v\left(s_{1}\right)\right)\right| \cdot \mid \int_{0}^{s_{1}} \frac{\left(s_{1}-u\right)^{(\beta-1)}}{\Gamma(\beta)} K(u, v(u), v(v(u))) d u \\
& -\int_{0}^{s_{2}} \frac{\left(s_{2}-u\right)^{(\beta-1)}}{\Gamma(\beta)} K(u, v(u), v(v(u))) d u \mid \\
& \leqslant N \cdot \ell \frac{\left|s_{1}-s_{2}\right| \beta}{\Gamma(\beta+1)}+n\left|v\left(s_{1}\right)-v\left(s_{2}\right)\right| \\
& +\left|f\left(s_{1}, v\left(s_{2}\right)\right)-f\left(s_{1}, v\left(s_{1}\right)\right)\right| \cdot \int_{0}^{s_{1}}\left|\frac{\left(s_{1}-u\right)^{(\beta-1)}}{\Gamma(\beta)} K(u, v(u), v(v(u)))\right| d u \\
& +\left|f\left(s_{1}, v\left(s_{1}\right)\right)-f\left(s_{2}, v\left(s_{1}\right)\right)\right| \cdot \int_{0}^{s_{1}}\left|\frac{\left(s_{1}-u\right)^{(\beta-1)}}{\Gamma(\beta)} K(u, v(u), v(v(u)))\right| d u \\
& +\left|f\left(s_{2}, v\left(s_{1}\right)\right)\right| \cdot \mid \int_{0}^{s_{1}} \frac{\left(s_{1}-u\right)^{(\beta-1)}}{\Gamma(\beta)} K(u, v(u), v(v(u))) d u \\
& -\int_{0}^{s_{2}} \frac{\left(s_{2}-u\right)^{(\beta-1)}}{\Gamma(\beta)} K(u, v(u), v(v(u))) d u \mid \\
& \leqslant(N+n) \cdot l \frac{\left|s_{1}-s_{2}\right|^{\beta}}{\Gamma(\beta+1)}+(M+m) \ell \cdot \frac{\left|s_{1}-s_{2}\right|^{\beta}}{\Gamma(\beta+1)} \cdot \int_{0}^{s_{1}}\left|\frac{\left(s_{1}-u\right)^{(\beta-1)}}{\Gamma(\beta)} k(u, v(u), v(v(u)))\right| d u \\
& +\left|f\left(s_{2}, v\left(s_{1}\right)\right)\right| \ell \frac{\left|s_{1}-s_{2}\right|^{\beta}}{\Gamma(\beta+1)} \\
& =\ell \frac{\left|s_{1}-s_{2}\right|^{\beta}}{\Gamma(\beta+1)}\left(\mathrm{N}+\mathrm{n}+(M+m) \cdot \int_{0}^{s_{1}}\left|\frac{\left(s_{1}-u\right)^{(\beta-1)}}{\Gamma(\beta)} K(u, v(u), v(v(u)))\right| d u+\left|f\left(s_{2}, v\left(s_{1}\right)\right)\right|\right) \\
& \leqslant \ell \frac{\left|s_{1}-s_{2}\right|^{\beta}}{\Gamma(\beta+1)} \text {. }
\end{aligned}
$$

Thus it follows that $\mathrm{G} v \in \mathrm{C}_{\ell, \beta}$, for all $v \in \mathrm{C}_{\ell, \beta}$. Then $\mathrm{T}: \in \mathrm{C}_{\ell, \beta} \rightarrow \in \mathrm{C}_{\ell, \beta}$.

As $s_{1} \rightarrow s_{2}$, the right hand side of the above inequality approaches zero. As a result of Phases 1 to 3 , using Arzelá-Ascoli theorem, we may deduce that $\mathrm{G}$ is completely continuous.

Phase 4. Beforehand bounds.

Presently, it remains to prove that the set

$$
\delta=\left\{v \in C_{\ell, \beta}: v=\rho G v \text { for some } 0 \leqslant \rho \leqslant 1\right\}
$$

is bounded.

Let $v \in \delta$, therefore $v=\rho G v$ for some $0 \leqslant \rho \leqslant 1$

$$
|v(s)| \leqslant \mid g\left(s, v(s)|+| f(s, v(s)) \cdot \int_{0}^{s} \frac{(s-u)^{(\beta-1)}}{\Gamma(\beta)} K(u, v(u), v(v(u))) d u \mid \leqslant \zeta+\Xi:=R .\right.
$$


According to the Schaefer's fixed point theorem $G$ has a fixed point that is a solution for equation (2.1).

In the next theorem, we will give the result for the existence of solution for the equation (2.1) by using the Schaefer's fixed point theorem.

Theorem 2.3. Suppose that $\left(a_{1}\right),\left(a_{3}\right),\left(a_{4}\right)$ in Theorem 2.2 are held and further

$\left(\check{a}_{2}\right)$ there is a constant $\check{\ell}$ so that

$$
|K(s, v(s), v(v(s)))-K(s, w(s), w(w(s)))| \leqslant \check{\ell}(\ell+2)|v(s)-w(s)|, \text { for eachs } \in \mathrm{I}, v, w \in R .
$$

If

$$
\sup _{s \geqslant 0}\left\{n+m \int_{0}^{s} \frac{(s-u)^{(\beta-1)}}{\Gamma(\beta)} K(u, v(u), v(v(u))) d u+\frac{s^{\beta}}{\Gamma(\beta+1)} \cdot|f(s, v(s))|\right\} \leqslant 1
$$

or

$$
\lim _{s \rightarrow \infty} \frac{s^{\beta}}{\Gamma(\beta+1)} .|f(s, v(s))|=0 \text { uniformly with respect to } v \in C_{\ell, \beta},
$$

then the equation (2.1) has at least one solution in $\mathrm{C}_{\ell, \beta}$.

Proof. Through the use of the Theorem 2.1 and Theorem 2.2, we prove that $\mathrm{G}$ is nonexpansive. By applying the Schaefer's fixed point theorem, we can deduce that $G$ has at least one fixed point which is used for solving the equation (2.1).

\section{Application}

We provide an example to interpret the utility of our major outcome. Consider the following nonlinear fractional iterative integral equation.

$$
v(s)=\frac{1}{s+9} \cdot v(s)+\cos (s(v(s))) \cdot \int_{0}^{s} \frac{(s-r)^{\frac{-1}{2}}}{\Gamma\left(\frac{1}{2}\right)} \cdot \frac{e^{-r}}{1+e^{r}} \cdot \frac{|v(v(r))|}{1+|v(v(r))|} d r .
$$

In this case, we get

$$
\begin{aligned}
g: R_{+} \times R \rightarrow R, g(s, v(s)) & =\frac{1}{s+9} \cdot v(s), \forall s \in I \\
f: R_{+} \times R \rightarrow R, f(s, v(s)) & =\cos (s v(s)), \forall s \in I .
\end{aligned}
$$

and

$$
\mathrm{K}: \mathrm{R}_{+} \times \mathrm{R} \times \mathrm{R} \rightarrow \mathrm{R}, \mathrm{K}(\mathrm{s}, v(\mathrm{~s}), v(v(\mathrm{~s})))=\frac{\mathrm{e}^{-\mathrm{s}}}{1+\mathrm{e}^{\mathrm{s}}} \cdot \frac{|v(v(\mathrm{~s}))|}{1+|v(v(s))|^{\prime}}, \forall \mathrm{s} \in \mathrm{I} .
$$

We are keen to study the solution $v$ subsidiary

$$
\mathrm{C}_{\ell, \beta}=\left\{v \in \mathrm{C}(\mathrm{I}, \mathrm{I}):\left|v\left(\mathrm{~s}_{1}\right)-v\left(\mathrm{~s}_{2}\right)\right| \leqslant \frac{\left|\mathrm{s}_{1}-\mathrm{s}_{2}\right|^{0.5}}{\Gamma(1.5)}=\frac{\left|\mathrm{s}_{1}-\mathrm{s}_{2}\right|^{0.5}}{0.8862269255}, \forall \mathrm{s}_{1}, \mathrm{~s}_{2} \in \mathrm{I}\right\}
$$

that means $\ell=1$. From Theorem 2.1 we get $\ell_{1}=\frac{1}{9}, \ell_{2}=\frac{1}{2}, \ell_{3}=\frac{2}{3}$.

If $\ell_{1}<k<1$ in Theorem 2.1, the equation (3.1) has a unique solution in I. From Theorem 2.2 we get

$$
\mathrm{n}=\frac{1}{9}, \mathrm{~N}=\frac{1}{9} \cdot \frac{\left(\frac{1}{4}\right)^{0.5}}{\Gamma(1.5)}=\frac{1}{16.838312}, \mathrm{~m}=\frac{1}{2}, \mathrm{M}=\frac{1}{2} \cdot \frac{1}{\Gamma(1.5)}=\frac{1}{1.7725}, \text { and } \Xi=\frac{1}{2 \Gamma(0.5)} .
$$

Also, from Theorem 2.3, we get $\breve{\ell}=\frac{2}{3}$. Therefore, equation (3.1) has at least one solution in $C_{1,0.5}$. 


\section{Conclusion}

From the above, we conclude that the fractional iterative differential equations are the best way to study the mathematical biological model and generally. Moreover, as future work, one can investigate the existence of solution for the following equations

$$
v(s)=g(s, v(s), v(v(s)))+f(s, v(s)) \int_{0}^{s} \frac{(s-u)^{(\beta-1)}}{\Gamma(\beta)} K(u, v(u), v(v(u))) d u
$$

and

$$
v(s)=g(s, v(s), v(v(s)))+f\left(s, v(s), v(v(s)) \int_{0}^{s} \frac{(s-u)^{(\beta-1)}}{\Gamma(\beta)} K(u, v(u), v(v(u))) d u\right.
$$

\section{Acknowledgment}

The authors would like to thank the reviewers for careful reading the paper and giving constructive comments, which improved the manuscript substantially. Further, the authors are also very grateful to University Putra Malaysia(UPM) for providing partial support to carry out this study.

\section{References}

[1] A. Atangana, R. T. Alqahtani, Numerical approximation of the space-time Caputo-Fabrizio fractional derivative and application to groundwater pollution equation, Adv. Difference Equ., 2016 (2016), 13 pages. 1

[2] A. Atangana, I. Koca, Chaos in a simple nonlinear system with Atangana-Baleanu derivatives with fractional order, Chaos Solitons Fractals, 89 (2016), 447-454. 1

[3] V. Berinde, Iterative approximation of fixed points, Second edition, Lecture Notes in Mathematics, Springer, Berlin, (2007). 1

[4] V. Berinde, Existence and approximation of solutions of some first order iterative differential equations, Miskolc Math. Notes, 11 (2010), 13-26. 2

[5] S. S. Cheng, J.-G. Si, X.-P. Wang, An existence theorem for iterative functional differential equations, Acta Math. Hungar., 94 (2002), 1-17. 1

[6] F. H. Damag, A. Kılıçman, R. A. A. Abdulghafor, Approximate solutions and existence result for some integral equation with modified argument of fractional order, Adv. Difference Equ., (2016), submitted. 1

[7] F. H. Damag, A. Kılıçman, R. W. Ibrahim, Approximate solutions for non-linear iterative fractional differential equations, Innovations Through Mathematical and Statistical Research, Proceedings of the 2nd International Conference on Mathematical Sciences and Statistics (ICMSS2016), AIP Publishing, 1739 (2016). 1

[8] F. H. Damag, A. Kılıçman, R. W. Ibrahim, Findings of fractional iterative differential equations involving first order derivative, Int. J. Appl. Comput. Math., (2016), 1-10. 1

[9] A. El-Sayed, H. Hashem, Existence results for nonlinear quadratic integral equations of fractional order in Banach algebra, Fract. Calc. Appl. Anal., 16 (2013), 816-826. 1

[10] A. Granas, J. Dugundji, Fixed point theory, Springer Science and Business Media, (2013). 1

[11] R. W. Ibrahim, A. Kılıçman, F. H. Damag, Existence and uniqueness for a class of iterative fractional differential equations, Adv. Difference Equ., 2015 (2015), 13 pages. 1

[12] R. W. Ibrahim, A. K1lıçman, F. H. Damag, Extremal solutions by monotone iterative technique for hybrid fractional differential equations, Turkish J. Anal. Number Theory, 4 (2016), 60-66. 1

[13] S. Ishikawa, Fixed points and iteration of a nonexpansive mapping in a Banach space, Proc. Amer. Math. Soc., 59 (1976), 65-71. 1

[14] M. Lauran, Existence results for some integral equation with modified argument, Gen. Math., 19 (2011), 85-92. 1

[15] M. Lauran, Existence results for some nonlinear integral equations, Miskolc Math. Notes, 13 (2012), 67-74. 1

[16] M. Lauran, Solution of first iterative differential equations, An. Univ. Craiova Ser. Mat. Inform, 40 (2013), 45-51. 1

[17] K. S. Miller, B. Ross, An introduction to the fractional calculus and fractional differential equations, A Wiley-Interscience Publication, John Wiley \& Sons, Inc., New York, (1993). 1

[18] D. O'Regan, Existence results for nonlinear integral equations, J. Math. Anal. Appl., 192 (1995), 705-726. 1

[19] I. Podlubny, Fractional differential equations, An introduction to fractional derivatives, fractional differential equations, to methods of their solution and some of their applications, Mathematics in Science and Engineering, Academic Press, Inc., San Diego, CA, (1999). 1

[20] S. G. Samko, A. A. Kilbas, O. I. Marichev, Fractional integrals and derivatives, Theory and applications, Edited and with a foreword by S. M. Nikolskiř, Translated from the 1987 Russian original, Revised by the authors, Gordon and Breach Science Publishers, Yverdon, (1993). 1 
[21] J.-R. Wang, M. Fečkan, Y. Zhou, Fractional order iterative functional differential equations with parameter, Appl. Math. Model., 37 (2013), 6055-6067. 1

[22] P.-P. Zhang, X.-B. Gong, Existence of solutions for iterative differential equations, Electron. J. Differential Equations, 2014 (2014), 10 pages. 1 